\title{
Remembrance of epigenomes past
}

\author{
Understanding of how epigenetic information is acquired, processed and transmitted through cell division, \\ and potentially across generations, remains limited. Mechanistic studies aiming to elucidate the molecular \\ underpinnings of these phenomena may provide insights into development, disease susceptibility and evolution.
}

0 ne of the pillars of genetics, and indeed biomedical research, is the notion that the heritable information required for cellular function is mainly encoded by DNA sequence. It is also widely documented that, during the course of embryonic development and throughout adulthood, as cells replicate, differentiate and age, the genome is dynamically decorated with epigenetic marks (for example, histone and DNA modifications) that correlate with different gene expression patterns, cell identity and potency. The canonical perspective has been that this epigenetic information is then erased in the germ line and is thus not inherited by the next generation. This allows the newly formed zygote to start its development with an epigenomic tabula rasa.

One area of intense research has focused on understanding how the early zygote departs from this seemingly blank state and transitions into a transcriptionally active state. Factors that initiate embryonic or zygotic genome activation (EGA or ZGA) have been reported in animals like fruit flies and zebrafish, but little is known about the identity of the 'master regulators' of this process in mammals.

Three studies now add a missing piece of the EGA puzzle, suggesting that the family of double-homeodomain (DUX) transcription factors is instrumental in unleashing the first wave of transcription in the embryos of of placental mammals. On page 925, Bradley Cairns and colleagues report that mouse Dux and human DUX4 are expressed before the onset of EGA and that they are activators of cleavage-stage genes and retroviral elements. Furthermore, they show that DUX expression can convert mouse embryonic stem cells (mESCs) into twocell-embryo-like (2C-like) cells in vitro. On page 941, Didier Trono and colleagues show that DUX depletion in mESCs affects the formation of 2C-like cells, while in embryos it impairs early development. Finally, on page 935, Stephen Tapscott and colleagues investigated the functional evolution of the DUX family in mouse and human cells. Their findings are also relevant for facioscapulohumeral dystrophy, which is caused by misexpression of DUX4 in skeletal muscle. Altogether, these reports deepen the knowledge about how the silence of the recently formed genome is broken and how new gene expression and chromatin patterns first emerge.

But what if the patterns of epigenetic information acquired during development or adulthood do not completely disappear in the germ line? What if new generations have a memory of epigenomes past? To address these questions, Giacomo Cavalli and colleagues ( $p$ 876) used a Drosophila transgenic line, continuously selecting flies with white or red eyes through several generations and thus establishing stable and isogenic lines carrying alternative epialleles (differentially marked by H3K27me3). Remarkably, they found that the epilines could be reset to a naive state and that the environment could modulate epiallele expressivity. This report, along with others using model organisms, contributes to a growing body of evidence supporting the existence of transgenerational epigenetic inheritance (TEI) in animals.

In humans, there are some epidemiological reports suggesting that TEI may exist, which has naturally stimulated popular imagination. However, numerous confounding factors in these studies and a lack of mechanistic explanations have precluded solid conclusions, and the field remains fraught with controversy.

While the evidence for genetic variation as the primary basis for trait and disease heritability is abundantly clear, it is important to ascertain whether other modes of inheritance exist and, if so, how frequent they are outside the laboratory. It remains to be seen whether the lessons learned from TEI studies with Drosophila and other animals are relevant to our species. Caution is warranted, but it is an intriguing hypothesis, and one that may yet help us better understand development, disease etiology and evolution. 